\title{
Learning Analytics e Computação Afetiva: Detecção da Frustração no Uso de um Jogo Educacional em Dispositivo Móvel
}

\section{Rogerio Bordignon, Viviane Ribeiro de Sousa, Yuri José dos Santos Serrano e Orlando Bisacchi Coelho}

Faculdade de Computação e Informática - Universidade Presbiteriana Mackenzie

orlandoc@mackenzie.br

\begin{abstract}
The identification of the affective state of a student allows the instructor to act in a way that facilitates learning. Frustration can be an important barrier to learning. This is an exploratory work aiming to use Learning Analytics to detect the onset of the frustration state when the student is involved in a learning task based on an educational game. Different personas were created presenting varying degrees of frustration and behavioural data was generated for five students corresponding to each persona. By analising this data it is possible to cluster the students in a way that the frustrated students can be identified.
\end{abstract}

Resumo: A identificação do estado afetivo do estudante pode permitir ao instrutor tomar ações que facilitem o aprendizado. A frustração pode ser uma barreira significativa para a aprendizagem. O trabalho aqui reportado é um estudo exploratório que se propôs a utilizar uma abordagem de Learning Analytics para detectar o início do estado de frustração quando o estudante realiza uma tarefa de aprendizado baseada em um jogo educacional. Foram criadas personas que simulam o comportamento de crianças com variados estados de frustração e gerados dados para cinco alunos correspondendo a cada persona. Analisando esses dados, pôde-se observar que é possível agrupar o conjunto de alunos de forma tal que se identifique os alunos frustrados. 
V Congresso Brasileiro de Informática na Educação (CBIE 2016)

Anais dos Workshops do V Congresso Brasileiro de Informática na Educação (CBIE 2016)

\section{Introdução}

Mobile learning (aprendizagem baseada em dispositivos móveis) é um paradigma que consiste no ensino e aprendizado através de dispositivos móveis, como smartphones e tablets, dentre outros, tendo como principal objetivo priorizar a mobilidade dos indivíduos e proporcionar um aprendizado mais dinâmico e flexível [ATTEWELL et. al 2005]. Dentro de certa faixa de idade, o ensino de lógica de programação tem-se mostrado uma abordagem efetiva para desenvolver o raciocínio lógico e as capacidades de resolução de problemas e de abstração [IEPSEN, BERCHT e REATEGUI 2013]. O uso de jogos para fins educacionais tem uma bem estabelecida base teórica de sustentação [RIEBER 1996; DEDE 2009]. No caso do ensino de crianças, em particular, devido ao fato de que o uso de tablets e smartphones é completamente natural para elas [MOURA 2009], o aprendizado com base em jogos que rodam em dispositivos móveis mostra-se uma opção bastante adequada.

Em qualquer processo de ensino-aprendizagem, a correta identificação do estado afetivo do estudante pode permitir que o instrutor tome ações que facilitem $o$ aprendizado. Isso torna-se ainda mais importante no contexto da aprendizagem mediada por sistemas computacionais [PICARD 1997]. Na área educacional identificar os estados afetivos das crianças pode permitir que ações sejam tomadas para auxiliá-los no andamento de suas atividades [PICARD 1997]. tendo em vista que a afetividade pode afetar o aprendizado de uma criança tanto negativamente quanto positivamente [BEHAR et al. 2013; LAGO 2011], fora a importância que a afetividade possui em processos cognitivos, memória e tomadas de decisão [PICARD 2000; PICARD 2010]. Segundo Picard e colegas (2004) uma das motivações para dar a sistemas computacionais características emocionais é fazer com que a interação homem-máquina seja menos frustrante.

A frustração é um estado afetivo resultante da ocorrência de um obstáculo que impede a satisfação de uma necessidade [LAWSON 1965]. Ela pode ser uma barreira significativa para a aprendizagem, visto o medo do fracasso que costumeiramente as pessoas possuem [SCHANK et al. 2001], podendo ser muito destrutiva ao ponto de levá-las a desistir da atividade que estão realizando [PICARD et al. 2004].

De modo a permitir que o sistema possa detectar o estado afetivo atual do aprendiz, a partir de uma sequência de ações comportamentais por ele efetuadas [QUARTO, OLIVEIRA e BERCHT 2014], o sistema tem de ser capaz de coletar uma série de dados de forma autônoma. Uma linha de trabalho extremamente interessante 
V Congresso Brasileiro de Informática na Educação (CBIE 2016)

Anais dos Workshops do V Congresso Brasileiro de Informática na Educação (CBIE 2016)

envolve a coleta de dados multimodais para posterior análise e detecção do estado emocional do aprendiz [D'MELLO, PICARD, GRAESSER 2007; KAPOOR, BURLESON e PICARD 2007]. Essa abordagem, entretanto, ainda não é prática e economicamente viável, dada a situação tecnológica atual. O trabalho aqui reportado propôs-se a utilizar somente os dados diretamente disponíveis a partir da própria execução da tarefa educacional em um dispositivo móvel, sem qualquer instrumentação adicional.

Uma vez coletados esses dados, o processo de fazer sentido deles e permitir compreender o eventual estado afetivo de início de frustração do aluno passa a ser uma tarefa de Mineração de Dados [WITTEN e FRANK 2005]. Mais precisamente, enquanto mineração de dados num contexto facilitador do processo de ensinoaprendizagem, uma tarefa de Learning Analytics [GAŠEVIC, DAWSON e SIEMENS 2015].

O trabalho aqui reportado refere-se a um estudo exploratório que se propôs a utilizar uma abordagem de Learning Analytics para detectar o início do estado de frustração quando o estudante realiza uma tarefa de aprendizado de lógica de programação. Para tal foi construído um jogo para ser executado em tablet, projetado para ensinar lógica de programação. Foram então criadas personas que simulam o comportamento de crianças na faixa etária de 07 a 12 anos durante o aprendizado de lógica de programação, com variações do estado de frustração entre as várias personas. Para cada persona foram gerados dados para 5 alunos distintos. Analisando os dados coletados a partir do comportamento desses alunos ao realizar a tarefa educacional, pôde-se observar que é possível agrupar o conjunto de alunos de forma tal que cada grupo corresponda precisamente a uma das personas. Esse estudo exploratório permitiu validar a abordagem baseada em Mineração de Dados e embasa o próximo experimento a ser realizado, dessa vez envolvendo crianças reais desempenhando a tarefa.

O presente trabalho está relacionado a outros trabalhos desenvolvidos no Brasil, tais como (IEPSEN, BERCHT e REATEGUI 2011) e (JAQUES set al. 2012), que focam na interação entre frustração e aprendizagem, mas ou não usam técnicas de mineração de dados ou não focam em agrupamento para tal. 
V Congresso Brasileiro de Informática na Educação (CBIE 2016)

Anais dos Workshops do V Congresso Brasileiro de Informática na Educação (CBIE 2016)

\section{Metodologia}

Foi desenvolvido um jogo em 2D para iPad para o ensino de lógica de programação, para alunos na faixa etária de 07 a 12 anos. A aplicação se fundamenta nos princípios da linguagem em blocos, baseada em quebra-cabeças. No jogo a criança deve utilizar os blocos disponíveis para levar o capitão Apólito às pilhas que darão energia para sua nave e assim voltar ao seu planeta de origem. $\mathrm{O}$ aplicativo possui um botão rotulado de ABORTAR; partiu-se da premissa de que quando um usuário utiliza tal botão, o mesmo se encontra próximo do estado de frustração, ou até mesmo frustrado. A criança pode apertá-lo a qualquer momento caso se sinta cansada ou chateada por não conseguir resolver o exercício.

Ao apertar esse botão ou conseguir terminar a fase, são coletadas informações como os padrões de toque, seu tempo de conclusão, quantidade de tentativas, tipo de toque e local do toque. Nesse momento, as crianças são redirecionadas para a próxima fase. O aplicativo conta com nove fases, que abordam conteúdos como: sequência lógica básica, laço de repetição e condicional. De modo a não existir uma escada de dificuldade muito inclinada, os assuntos são trabalhados de forma que, em um grupo de três fases, cada fase se diferencia entre os temas citados. Assim, nessas nove fases temos três níveis de dificuldade: fácil, para as fases um a três; médio, para as etapas quatro a seis; e difícil, para o restante. Quando o usuário acerta a resposta, movimentamos o Capitão Apólito até a pilha para que seja perceptível e gratificante a conclusão de um exercício. Logo após, uma mensagem aparece dando-lhe os parabéns. Ao final de cada fase todos os dados são enviados para um servidor, para posterior préprocessamento e análise. A cada fase jogada salvamos a seguinte série de dados de uso do jogador.

I. Fase: a fase atual do jogo.

II. Aluno: um id gerado automaticamente para cada aluno, a partir da fase um.

III. Tempo: o tempo que o usuário levou para concluir a fase, em milisegundos;

IV. Tentativas: a quantidade de tentativas durante a fase;

V. Frustrado: um campo booleano, que é verdadeiro caso o usuário tenha pressionado o botão abortar para pular a fase atual e

VI. Toques: todos os toques que foram dados na tela, contendo: a posição na tela, dada pelas coordenadas $\mathrm{X}$ e $\mathrm{Y}$, o momento no tempo T em que foi dado o toque e a ação 
V Congresso Brasileiro de Informática na Educação (CBIE 2016)

Anais dos Workshops do V Congresso Brasileiro de Informática na Educação (CBIE 2016)

que foi realizada, codificada por um valor de 0 a 12. Essas ações realizadas pelo aluno podem ser: Lugar sem Ação, Avance, Direita, Esquerda, Condicional Se, Laço Repita, Abortar, Sim após o Abortar, Não, após o Abortar, Executar, Limpar e a Mensagem de Acerto gerada pelo sistema.

O pré-processamento consistiu em contar a quantidade de toques no array de toques e então adicionar ao arquivo um campo numerado sequencialmente para cada toque. Também foram criados os campos: quantidades de toque total da fase, média de toques por segundo; e, além disso, os campos correspondentes às quantidades de toques de cada um dos tipos descritos no parágrafo acima.

Com base no comportamento observado em um ambiente de ensino de programação a alunos dessa faixa etária, foram então criadas quatro personas.

I. Persona A: correspondente a alunos que não apresentam dificuldades, completam todas as fases sem utilizar o botão de frustração e cometem alguns erros apenas, por falta de atenção.

II. Persona B: correspondente a alunos que tem uma certa dificuldade, mas após utilizar uma quantidade menor do que 6 tentativas completaram os exercícios. Julgamos que 6 é um valor adequado pois é o dobro da média de toques correspondentes à Persona $\mathrm{A}$.

III. Persona C: refere-se a alunos que se frustraram nos exercícios mais difíceis, mas que fizeram grandes quantidades de tentativas.

IV. Persona D: corresponde a alunos que ficaram desmotivados em poucas tentativas e acabaram mudando de fase sem um real aprendizado, caracterizando-se assim o estado emocional de frustração (SCHANK et al., 2001).

Foram simulados os comportamentos de vinte crianças; cinco alunos correspondendo a cada uma das personas descritas anteriormente. A Persona A corresponde aos alunos numerados de $1 \mathrm{a} 5$. A Persona B corresponde aos alunos de 6 a 10. A Persona C corresponde aos alunos de 11 a 15. E a Persona D corresponde aos alunos de 16 a 20.

O objetivo do experimento exploratório aqui reportado foi identificar se, a partir do comportamento dos dados gerados por cada estudante simulado, seria possível agrupar corretamente os vinte estudantes nas quatro personas. Para isso fui utilizado o algoritmo K-Médias, tal como implementado no software Weka [WITTEN e FRANK 2005]. 
V Congresso Brasileiro de Informática na Educação (CBIE 2016)

Anais dos Workshops do V Congresso Brasileiro de Informática na Educação (CBIE 2016)

Agrupamento é uma tarefa não supervisionada que se propõe a segmentar os dados em grupos (clusters), aproximando dados similares, tendo em vista que os elementos de um grupo sejam semelhantes entre si e diferentes dos elementos dos outros grupos. K-Médias é um algoritmo de agrupamento bastante robusto. Ele objetiva minimizar a distância dos elementos em relação a um conjunto de $\mathrm{K}$ centros de forma iterativa, tendo em vista que a distância entre um ponto e um conjunto de clusters é definida como sendo a distância do ponto ao centro mais próximo dele [MACKAY 2003].

Como no experimento foram sintetizados dados para 4 personas de diferentes comportamentos, usamos o parâmetro $\mathrm{K}$ do K-Médias com o valor 4, buscando observar se os resultados do agrupamento identificavam corretamente as quatro personas.

\section{Resultados e Discussão}

Após a captação e análise, chegamos aos valores demonstrados na Tabela 1 para cada fase trabalhada e a taxa de exatidão no agrupamento de cada persona.

Tabela 1. Resultados da captação de dados em cada fase e sua taxa de exatidão no agrupamento.

\begin{tabular}{|c|c|c|c|c|c|}
\hline Fase & $\begin{array}{r}\text { Média de } \\
\text { Tentativas }\end{array}$ & $\begin{array}{c}\text { Quantidade de } \\
\text { Frustrados }\end{array}$ & $\begin{array}{c}\text { Média de Toques } \\
\text { por Segundo }\end{array}$ & $\begin{array}{c}\text { Média de Toques } \\
\text { do Tipo 0 }\end{array}$ & $\begin{array}{r}\text { Taxa de } \\
\text { Exatidão }\end{array}$ \\
\hline 1 & 3,50 & 9 & 1,50 & 8,80 & $95 \%$ \\
\hline 2 & 3,45 & 9 & 1,40 & 11,50 & $70 \%$ \\
\hline 3 & 3,55 & 9 & 1,52 & 7,45 & $95 \%$ \\
\hline 4 & 3,45 & 9 & 1,44 & 9,50 & $95 \%$ \\
\hline 5 & 3,85 & 10 & 1,55 & 10,65 & $90 \%$ \\
\hline 6 & 3,45 & 10 & 1,52 & 10,25 & $100 \%$ \\
\hline 7 & 3,45 & 10 & 1,67 & 12,95 & $95 \%$ \\
\hline 8 & 3,50 & 10 & 1,53 & 10,75 & $85 \%$ \\
\hline 9 & 4,20 & 10 & 1,57 & 15,10 & $95 \%$ \\
\hline
\end{tabular}


V Congresso Brasileiro de Informática na Educação (CBIE 2016)

Anais dos Workshops do V Congresso Brasileiro de Informática na Educação (CBIE 2016)

Das nove fases analisadas pelo algoritmo, uma conseguiu separar os clusters de acordo com as personas com 100\% de acerto; cinco tiveram $95 \%$ de acerto na separação das personas; uma teve $90 \%$ e as restantes tiveram $85 \%$ e $70 \%$ de acerto.

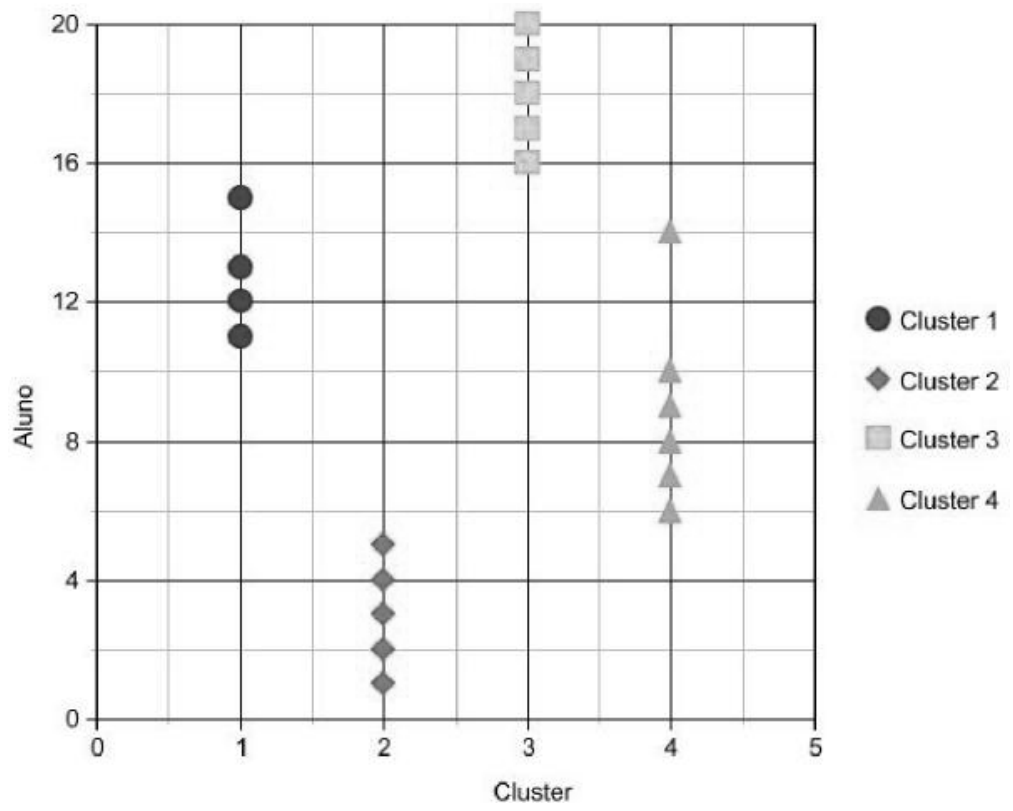

Figura 1. Resultado do algoritmo K-Médias para a fase um.

Na Figura 1, que mostra as informações dos clusters criados após o algoritmo agrupar a fase um do jogo, observamos que dos quatro grupos criados, houve acerto na identificação de 95\% das personas criadas. De fato, podemos constatar que: a Persona A é representada pelo cluster número 2; a Persona $\mathrm{B}$ corresponde ao cluster número 3; a Persona $\mathrm{C}$ é representada pelo cluster número 1 e a Persona $\mathrm{D}$ é representada pelo cluster número 4. Por outro lado, pode-se notar que o aluno de número 14, correspondendo à persona $\mathrm{C}$, foi agrupado com os alunos correspondentes à Persona $\mathrm{B}$. Entretanto, esse erro no agrupamento é razoável, devido ao fato de que ambas as personas foram simuladas com características semelhantes, como alta quantidade de toques e quantidade similar de tentativas. Pequenos erros no agrupamento, tal como o reportado aqui, também ocorreram em outras fases do jogo.

A Figura 2 apresenta o resultado do algoritmo para a fase oito, no qual se percebe que houve uma mistura entre as personas $\mathrm{C}$ e $\mathrm{D}$ nos clusters número 3 e 1 . Pois em ambos os grupos existem personas com propensão ao desenvolvimento da frustração e, dentre dez alunos, apenas um não apertou o botão abortar e concluiu a fase. Então ao calcular os centróides, ambos os clusters têm o dado abortou como verdadeiro; mas por 
V Congresso Brasileiro de Informática na Educação (CBIE 2016)

Anais dos Workshops do V Congresso Brasileiro de Informática na Educação (CBIE 2016)

projeto, a persona $\mathrm{D}$ possui uma quantidade de tentativas e tempo bem menor que a persona $\mathrm{C}$.

Porém, nessa fase, o aluno quinze, como mostrado na Tabela 2, teve uma quantidade de toques e tentativas um pouco mais alta que o comum, fazendo com que os centróides do cluster número 3 ficassem com uma média mais alta nesses atributos; fato esse que corroborou para que três alunos ficassem no agrupamento errado, de tal maneira que o cluster número 1 só foi formado porque decidimos previamente que o parâmetro $\mathrm{K}$ (que corresponde ao número de agrupamentos) desejado, no algoritmo K-Médias como sendo quatro, o que forçou a separação dos alunos treze e quatorze em um cluster muito semelhante ao terceiro.

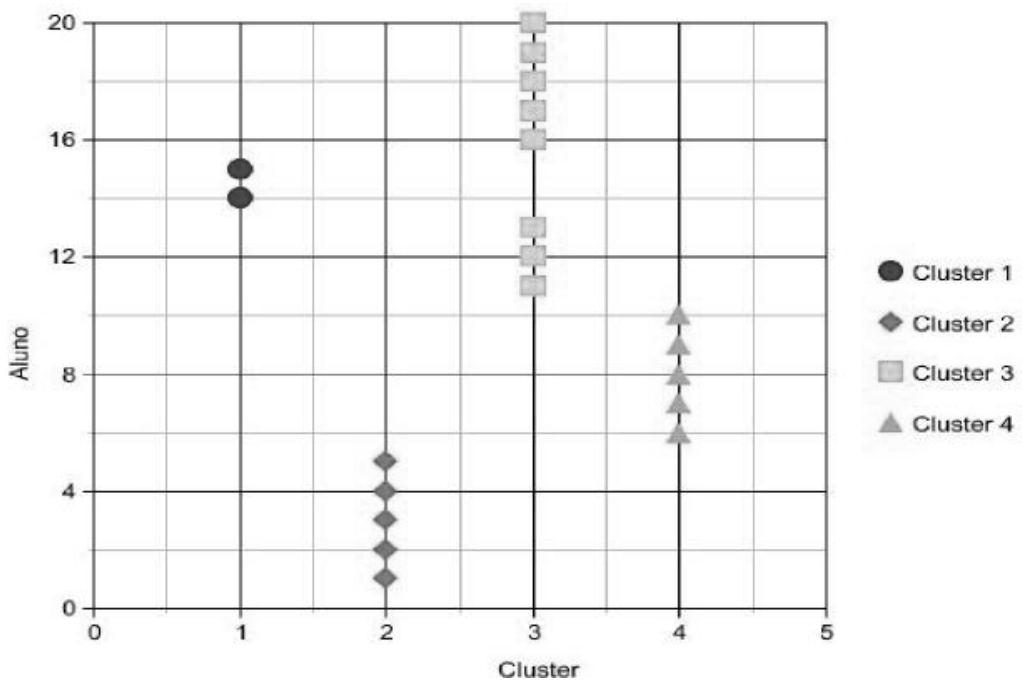

Figura 2. Resultado do algoritmo K-Médias para a fase oito.

Tabela 2. Dados dos alunos destacados na fase oito.

\begin{tabular}{|c|c|c|c|}
\hline Aluno & Quantidade de Toques & Tempo Total & $\begin{array}{c}\text { Média de Toques } \\
\text { por Segundo }\end{array}$ \\
\hline 10 & 41 & 31,0 & 1,3 \\
\hline 11 & 81 & 26,0 & 3,1 \\
\hline 12 & 68 & 40,0 & 1,7 \\
\hline 13 & 45 & 51,5 & 0,9 \\
\hline 14 & 82 & 68,5 & 1,2 \\
\hline 15 & 90 & 65,4 & 1,4 \\
\hline
\end{tabular}


V Congresso Brasileiro de Informática na Educação (CBIE 2016)

Anais dos Workshops do V Congresso Brasileiro de Informática na Educação (CBIE 2016)

\section{Conclusão}

O objetivo deste trabalho foi identificar o início do sentimento de frustração de crianças ao realizarem uma tarefa educacional, o que permitiria ao professor atuar para que um estudante não desista da atividade proposta por causa de sua frustração com a mesma. Desenvolvemos um aplicativo para o ensino de lógica de programação, utilizando conceitos da Computação Afetiva, combinados com técnicas de Mineração de Dados, que objetivava identificar quatro perfis de alunos previamente estabelecidos, que apresentavam graus diversos de frustração. $O$ experimento mostrou-se bem-sucedido, salvo um pequeno número de erros no agrupamento dos alunos correspondentes a cada perfil.

O próximo passo na pesquisa é validar o aplicativo num contexto real de ensino de programação a crianças na faixa etária de 07 a 12 anos. Certamente os dados obtidos de crianças serão muito mais ricos do que os dados sintéticos usados no experimento aqui reportado. Pretendemos usar algumas outras técnicas de agrupamento, além do K-Médias, tais como K-means++ (ARTHUR e VASSILVITSKII 2007) e X-means (PELLEG e MOORE 2000).

Temos ainda o propósito de desenvolver um sistema de recomendação da ação que deve ser tomada ao se detectar que o aluno apresenta os primeiros sinais de frustração. Além de buscar a eventual identificação de mais estados emocionais que podem influenciar no desempenho do estudante.

\section{Referências Bibliográficas}

ARTHUR, David, VASSILVITSKII, Sergei. k-means++: The Advantages of Careful Seeding. ACM-SIAM Symposium on Discrete Algorithms (SODA 2007). New Orleans, de 7 a 9 de janeiro, 2007.

BEHAR, Patricia A. et al. (2013) Competências em Educação a Distância, Porto Alegre: Penso.

D'MELLO, Sidney, PICARD, Rosalind e GRAESSER, Arthur. Towards an AffectSensitive AutoTutor. IEEE Intelligent Systems, 22(4), p. 53-61, 2007.

GAŠEVIĆ, Dragan; DAWSON, Shane; SIEMENS, George. Let's not forget: Learning analytics are about learning. TechTrends, v. 59 (1), p. 64-67, 2015. 
DEDE, Chris. Immersive Interfaces for Engagement and Learning. Science, v. 323 (5910), pp. 66-69, 2009.

IEPSEN, Edécio F., BERCHT, M., REATEGUI, E. Avaliando a Dimensão Afetiva para Apoio ao Processo de Aprendizagem na Disciplina de Algoritmos: um Estudo de Caso. Revista Latinoamericana de Tecnología Educativa, v. 12, p. 55-66, 2013.

JAQUES, Patricia A., Nunes, Maria Augusta S. N., Bittencourt, Ig. Computação Afetiva aplicada à Educação: Dotando Sistemas Tutores Inteligentes de Habilidades Sociais. I Workshop de Desafios da Computação Aplicada à Educação (DesafIE). Curitiba, de 16 a 19 de julho de 2012.

KAPOOR, Ashish, BURLESON, Winslow, PICARD, Rosalind W. Automatic prediction of frustration. International Journal of Human-Computer Studies, v. 65, pp. 724-736, 2007.

LAGO, N. A. Afetividade e emoções no ensino/aprendizagem de línguas: múltiplos olhares. Campinas: Pontes Editores, 2011.

LAWSON, P. R. Frustration: The development of a scientific concept. New York, USA: Macmillan, 1965.

MACKAY, David. Information Theory, Inference and Learning Algorithms. Cambridge University Press, 2003.

MOURA, Adelina. Geração Móvel: um ambiente de aprendizagem suportado por tecnologias móveis para a "Geração Polegar". Braga: Universidade do Minho, 2009, p.50-78.

PELlEG, Dan, MOORE, Andrew. X-means: Extending K-means with Efficient Estimation of the Number of Clusters. Proceedings of the Seventeenth International Conference on Machine Learning (CML'00). Stanford, 2000.

PICARD, Rosalind W. Affective Computing. Cambridge: MIT Press. USA, 1997.

PICARD. Rosalind. W . Affective Computing: from Laughter to IEEE. IEEE Transaction Education on Affective Computing, vol. 1, No. 1, p.11-17, 2010.

PICARD, Rosalind W.; PAPERT, S.; BENDER, W.; BLUMBERG, B.; BREAZEAL, C.; CAVAllO, D.; MACHOVER, T.; RESNICK, M.; Roy, D.; Strohecker, C. Affective learning — a manifesto. BT Technology Journal, 2004.

QUARTO, Cícero C., OLIVEIRA, Antônio N. e BERCHT Magda. Agentes Pedagógicos com Dimensões Afetivas: uma tecnologia no apoio ao ensino e a aprendizagem. XXV Simpósio Brasileiro de Informática na Educação (SBIE 2014). Dourados, de 03 a 06 de novembro de 2014.

RIEBER, Lloyd P. Seriously considering play: Designing interactive learning environments based on the blending of microworlds, simulations, and games. Educational Technology Research and Development, v. 44(2), p. 43-58, 1996.

SCHANK, R.; NEAMAN, A. Motivation and failure in educational systems design. AAAI Press and MIT Press, Cambridge, 2001.

WITTEN Iain. H; FRANK, Eibe. Data Mining - Practical Machine Learning Tools and Techniques. Elsevier, 2005. 